\title{
Type II collagen fragment HELIX-II is a marker for early cartilage lesions but does not predict the progression of cartilage destruction in human knee joint synovial fluid
}

\author{
Xiaochun Wei $\cdot$ Kun Yin $\cdot$ Pengcui Li $\cdot$ Huan Wang $\cdot$ \\ Juan Ding · Wangping Duan $\cdot$ Lei Wei
}

Received: 7 September 2011/Accepted: 10 December 2011/Published online: 12 January 2012

(C) The Author(s) 2012. This article is published with open access at Springerlink.com

\begin{abstract}
To determine whether there is a direct correlation between the concentration of type II collagen fragment HELIX-II in synovial fluid and the severity of cartilage damage at the knee joint, 83 patients who had undergone knee arthroscopy or total knee replacement were enrolled in this study (49\% women, mean \pm SD age $49.5 \pm 19$ ). The content of HELIX-II in the synovial fluid samples was measured by enzyme-linked immunosorbent assay (ELISA). Cartilage damage at the knee joint was classified during arthroscopy or direct surgical observation, using the Outerbridge cartilage damage scoring system. The maximum damage score was defined as the highest score among the six areas of the knee joint, and the cumulative score was defined as the sum of the scores of the six areas of the knee joint. The intra-assay and interassay variations of the HELIX-II ELISA were lower than 13 and $15 \%$, respectively. The level of HELIX-II in the severely damaged cartilage groups (cumulative scores $=$ 11-24 or maximum score $=2-4$ ) was much higher than
\end{abstract}

\section{Wei $(\bowtie) \cdot$ L. Wei $(\bowtie)$}

Department of Orthopaedics, The Second Hospital of Shanxi

Medical University, 382 Wuyi Road, Taiyuan 030001,

Shanxi, People's Republic of China

e-mail: weixiaochun08@126.com

L. Wei

e-mail: Lei_Wei@brown.edu

X. Wei · L. Wei

Shanxi Key Lab of Bone and Soft Tissue Injury Repair, 382 Wuyi Road, Taiyuan 030001, Shanxi,

People's Republic of China

K. Yin - P. Li - H. Wang - J. Ding - W. Duan - L. Wei Department of Orthopaedics, Rhode Island Hospital,

The Warren Alpert Medical School of Brown University, Suite 402A, 1 Hoppin Street, Providence, RI 02903, USA in the slightly damaged cartilage groups (cumulative scores $=0-10$ or maximum score $=0-1)$. The level of HELIX-II in cartilage from severely damaged cartilage groups was significantly higher than in the slightly damaged groups, but no significant difference was detected in the level of HELIX-II among the severely damaged cartilage sub-groups. There was a significant correlation between the HELIX-II concentration in the synovial fluid and the cumulative $(r=0.807)$ and maximum scores $(r=0.794)$. Thus, elevated HELIX-II level is correlated with early cartilage lesions, but does not have the sensitivity to predict the progression of severity of cartilage damage in the knee joint.

Keywords HELIX-II · Human synovial fluid . Cartilage damage $\cdot$ Biomarker

\section{Introduction}

Osteoarthritis (OA) is the most common cause of joint pain and disability in the world and is characterized by the progressive damage of articular cartilage [1, 2]. Early diagnosis is crucial for the prevention of and treatment for OA. Currently, plain X-rays have been the primary imaging method used to confirm the diagnosis of OA (when an $\mathrm{X}$ ray shows narrowed joint space due to cartilage loss), stage its severity, and follow its progression. Unfortunately, the severity of radiographic findings does not correlate well with the severity of symptoms, nor with functional disability [3,4]. These shortcomings limit the use of radiography both as a diagnostic tool and as a tool for ongoing assessment of disease progression. With the use of sensitive assays to recognize biomarkers in body fluids, researchers could identify early signs of disease and 
standardize the measures of progression, which could then increase the predictive power needed for clinical trials of new treatments [5]. As the alteration in cartilage matrix turnover occurs at the early stage of the disease, it is of great importance to find the biomarkers for early diagnosis of OA.

During the biological turnover of cartilage, some metabolites are released into the serum and synovial fluid [6]. In the case of damage or trauma, the damaged cartilage cells will also release lysosomal enzymes and collagenase, which can lead to the degradation of type II collagen [7-9]. Because these metabolites and degradation products change rapidly and dynamically with cartilage damage, they could be used as specific biomarkers reflecting alterations in the metabolism of cartilage [10-12].

Type II collagen is the main extracellular matrix protein in hyaline articular cartilage. Degradation of type II collagen is one of the metabolism alterations that take place with cartilage damage. Measuring type II collagen metabolites in the fluid surrounding an articular joint would therefore be a specific indicator of cartilage degradation at that joint. One of such metabolites is type II collagen helical peptide (HELIX-II), a molecule originating from the helical region of type II collagen.

Several studies have demonstrated that the presence of HELIX-II in serum or urine samples is positively associated with cartilage damage [13-15], but there is no direct evidence to demonstrate this relationship. In this study, we measured the level of HELIX-II in the knee joint's synovial fluid by ELISA, identified cartilage damage by arthroscopic findings, and classified it using the Outerbridge scoring system. We found that the level of HELIX-II in the synovial fluid from the knee joint correlates with early lesions in associated knee's articular cartilage, but it is not sensitive enough to predict the progression of cartilage damage.

\section{Materials and methods}

Enrollment of patients

The study enrolled 83 patients ( 42 men and 41 women) with an average age of 49.5 years $(49.5 \pm 19)$. Among this patient cohort, 42 received total knee replacement because of osteoarthritis and 41 underwent arthroscopy because of meniscus injury $(n=25)$, anterior or posterior cruciate ligament injury $(n=14)$, loose body $(n=1)$, or patellar dislocation $(n=1)$. Patients who had inflammatory joint disease, acute major trauma, malignant tumors, or abnormal renal and liver function were excluded from the study. Patients who took corticosteroid treatment within the 3 months preceding surgery were also excluded from the study.

\section{Collection and storage of synovial fluid}

A volume of $0.5-5 \mathrm{ml}$ of synovial fluid was aspirated from the knee joint just before total knee replacement or arthroscopy. The synovial fluid was immediately centrifuged for $15 \mathrm{~min}$ at $3,000 \mathrm{rpm}$ to remove particulate material and any clots, and the supernatants were aliquoted, rapidly frozen, and stored at $-70^{\circ} \mathrm{C}$.

\section{Evaluation of cartilage damage}

Articular cartilage lesions were evaluated during arthroscopy or via direct surgical observation, using the Outerbridge scoring system [19]. The absence of morphological changes in the articular cartilage is regarded as 0 points in the Outerbridge scoring system, and softened or swollen articular cartilage is given 1 point. Once fissures smaller than $1.5 \mathrm{~cm}$ appear on the articular cartilage surface, a score of 2 points is given, while fissures larger that $1.5 \mathrm{~cm}$ merit a score of 3 points. When cartilage flakes are detached from the articular surface, and the subchondral bones are exposed, the Outerbridge score is 4 points [19].

Each of the six regions of the knee cartilage (i.e., patellar, femoral groove, medial femoral condyle, lateral femoral condyle, medial tibial plateau, and lateral tibial plateau) was given an Outerbridge score (Fig. 1). The highest score recorded within one knee joint was defined as the maximum score, and the addition of all six scores within one knee joint was defined as the cumulative score. Therefore, the highest possible maximum and cumulative scores were 4 and 24 , respectively.

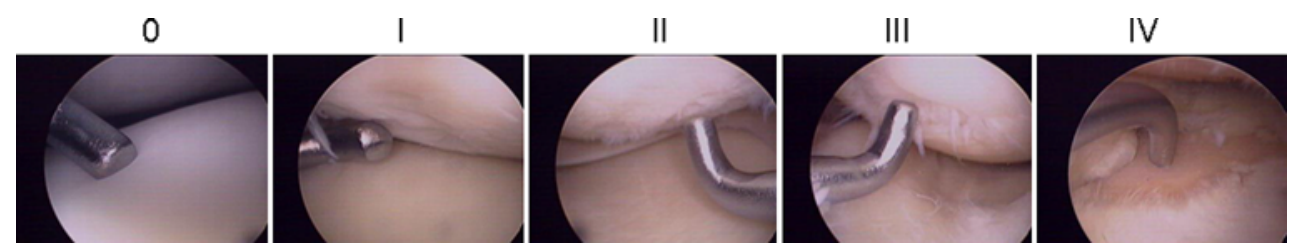

Fig. 1 Lesion of knee articular cartilage evaluated by arthroscopy with Outerbridge score system. 0 points no morphological changes in the cartilage, 1 point cartilage softened or swollen, 2 points fissures forming or already formed, but less than $1.5 \mathrm{~cm}, 3$ points fissures larger than $1.5 \mathrm{~cm}, 4$ points subchondral bone exposed or cartilage flakes detached 
Measurement of synovial HELIX-II

Synovial fluid HELIX-II content was measured using a competitive polyclonal antibody-based ELISA (enzymelinked immunosorbent assay) kit (Rongweida Corporation, Shanghai, China). After thawed to room temperature, all samples were diluted at a ratio of $1: 1 ; 50$ ul of the diluted sample and $50 \mathrm{ul}$ of biotinylated anti-HELIX-II antibody were then added to each well of a 96-well plate. After $1 \mathrm{~h}$ of incubation at $37^{\circ} \mathrm{C}$, the wells were drained and washed 3 times with washing buffer; $80 \mathrm{ul}$ of streptavidin-HRP solution was then added to the wells, and the plate was incubated for $30 \mathrm{~min}$ at $37^{\circ} \mathrm{C}$, and $50 \mathrm{ul}$ of the kit's Substrate A and Substrate B was added to each well, followed by incubation for $10 \mathrm{~min}$ at $37^{\circ} \mathrm{C}$ before the reaction was stopped by $50 \mathrm{ul}$ of $\mathrm{H}_{2} \mathrm{SO}_{4}$. The absorbance at each well was read by a spectrophotometer (The Packard FluoroCount BF10000) at $450 \mathrm{~nm}$ wavelength. All samples were measured as triplets, and the average reading was recorded.

\section{Statistical analyses}

All data are expressed as mean $\pm \mathrm{SD}$ and analyzed using SPSS 13.0 software (SPSS Inc, Chicago, IL). Student's $T$ test was used for paired group comparisons, and one-way ANOVA was used for inter-group comparisons. Nonparametric Spearman's rank correlation coefficient was used for correlation analysis. $P$ values less than 0.05 were considered statistically significant.

\section{Results}

HELIX-II concentration in synovial fluid was dependent on cartilage damage as determined by the accumulative Outerbridge score

We found that the 83 patients could be divided into two sub-population groups based on their cumulative Outerbridge score and their concentration of HELIX-II in synovial fluid (Fig. 2). Thirty-five patients had cumulative Outerbridge score less than 10 , and these will be referred to as the "slightly damaged cartilage" group. The remaining 48 patients had cumulative Outerbridge scores higher than 10 and will be referred to as the "severely damaged cartilage" group. The analysis showed that the HELIX-II content in the synovial fluid of the patients from the severely damaged cartilage group $(1.11 \pm 0.23 \mathrm{nmol} / \mathrm{l})$ was much higher than that of the slightly damaged cartilage group $(0.54 \pm 0.19 \mathrm{nmol} / \mathrm{l})$, and this difference was statistically significant $(P<0.0001)$. A significant correlation was found between the HELIX-II concentration in synovial fluid and the cumulative scores $(r=0.807)$.

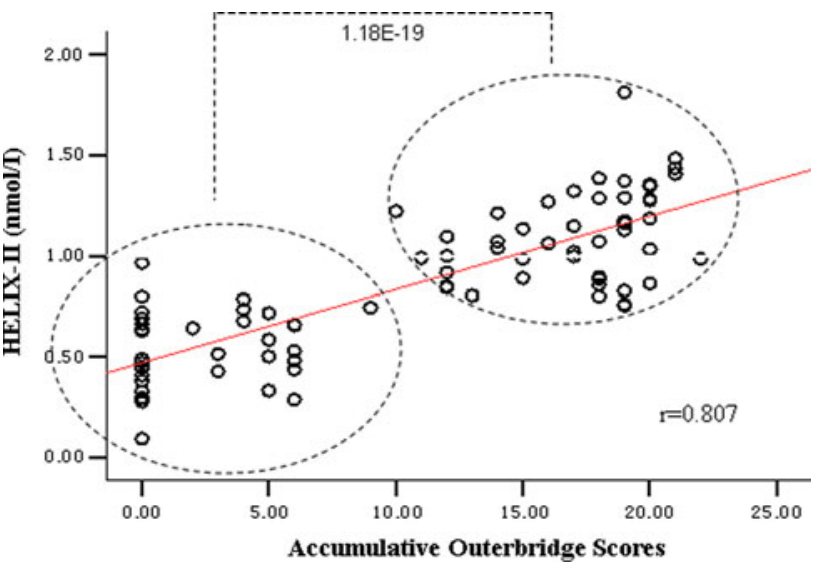

Fig. 2 The level of HELIX-II directly correlates with the cumulative Outerbridge score. Mean concentrations of HELIX-II in the synovial fluid significantly increased in the severely damaged cartilage group (cumulative Outerbridge score 11-24) in comparison with the slightly damaged cartilage group (cumulative Outerbridge score 1-10). There was a significant correlation $(r=0.807)$ between the HELIX-II concentration in synovial fluid and the articular cartilage cumulative Outerbridge score

HELIX-II concentrations in synovial fluid were correlated with cartilage damage as determined by the maximum Outerbridge score

We found that the 83 cases could also be divided into 5 groups based on their maximum Outerbridge score. Only a relatively small amount of HELIX-II was found in the synovial fluid of knee joints with maximum Outerbridge scores between 0 and 1 (slightly damaged cartilage groups). The average HELIX-II concentration in synovial fluid from the joints with a maximum Outerbridge score of 0 was $0.51 \pm 0.22 \mathrm{nmol} / \mathrm{l}$, and for those with a maximum Outerbridge score of 1 , it was $0.56 \pm 0.15 \mathrm{nmol} / \mathrm{l}$. There was no significant difference detected in the synovial fluid's HELIX-II concentration of knee joints with a maximum Outerbridge score of 0 and $1(P>0.386)$. However, the mean concentration of HELIX-II in the synovial fluid increased dramatically in knees with Outerbridge scores 2-4 (severely damaged cartilage groups), when compared with the slightly damaged cartilage groups $(P<0.0001)$. Maximum Outerbridge scores of 2,3 , and 4 were associated with mean HELIX-II concentrations of $0.97 \pm 0.16, \quad 1.06 \pm 0.15, \quad$ and $\quad 1.16 \pm 0.26 \mathrm{nmol} / \mathrm{l}$, respectively (Fig. 3). A significant correlation was found between the HELIX-II concentration in synovial fluid and the maximum scores $(r=0.794)$ (Fig. 3).

The average content of HELIX-II in the severely damaged cartilage group was $1.10 \pm 0.23 \mathrm{nmol} / \mathrm{l}$, which was much higher than that in the slightly damage group, which had $0.53 \pm 0.19 \mathrm{nmol} / 1(P<0.0001)$. This suggests that the concentration of synovial HELIX-II increases with cartilage damage. Interestingly, inter-group comparisons 


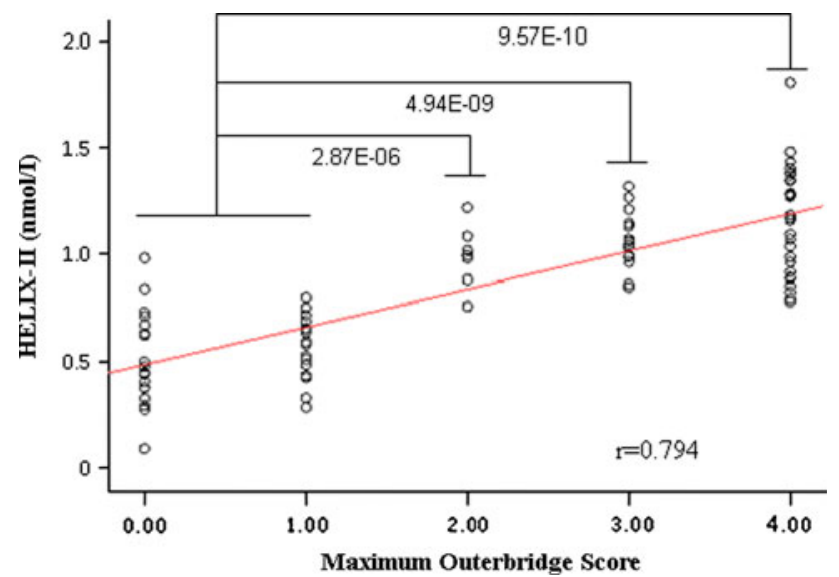

Fig. 3 The level of HELIX-II directly correlated with the maximum Outerbridge score. Mean concentrations of HELIX-II in the synovial fluid were significantly elevated between the slightly cartilage damage group (maximum Outerbridge scores $0-1$ ) and the severely damaged cartilage group (maximum Outerbridge scores 2-4). However, there was no significance difference in HELIX-II concentration among the severely damaged cartilage sub-groups (maximum Outerbridge score $2-4)$. There was a significant correlation $(r=0.794)$ between the HELIX-II concentration in synovial fluid and the articular cartilage maximum Outerbridge score

did not detect significant differences in the HELIX-II content among the severely damaged cartilage sub-groups (Maximum Outerbridge scores 2, 3 and 4) (Fig. 3), although there was an increasing trend. The mean concentration of HELIX-II only increased by $7.8 \%$ in the maximum score 3 sub-group as compared to the maximum score 2 sub-group $(P=0.2596)$. The mean concentration increased by $8.6 \%$ in the maximum score 4 sub-group relative to the maximum score 3 sub-group $(P=0.2279)$ (Fig. 3).

\section{Discussion}

Unlike most tissues in the body, articular cartilage is an avascular tissue with poor capacity for self-repair. Most articular cartilage injuries are maintained for years and can eventually lead to further damage. Therefore, early detection would be essential for the diagnosis, treatment, and prevention of cartilage damage. Radiographic assessment of joint space narrowing (an indicator of cartilage loss) is the only validated measure of OA progression; however, it is unable to detect early cartilage damage, nor has it been shown to correlate with symptoms or functional disability $[3,4,16]$. Assays that recognize specific biomarkers of cartilage metabolism in body fluids could potentially be used to detect early cartilage damage.

Type II collagen is the main extracellular matrix protein of hyaline articular cartilage. The metabolites or degradation products of type II collagen could be used as biomarkers to indicate the alterations in the metabolism of articular cartilage [2, 10-12, 17]. Most available studies report using serum or urine samples for the detection of biomarkers of articular cartilage degradation [13-15]. As type II collagen is not only resident in the articular cartilage, but also in other tissues such as nucleus of intervertebral disks, the levels of its metabolites or degradation products in serum frequently do not correlate with structural changes and may not accurately reflect changes on the articular cartilage [18]. The synovial fluid exists in the cavity of joints, where it provides internal nutrition to the articular cartilage. Theoretically, biomarkers in the synovial fluid of joints affected with OA could directly reflect the pathological changes in articular cartilage with less interference factors compared with serum or urine.

The Outerbridge scoring system has been used to evaluate cartilage damage for a long time. A recent study demonstrated that orthopedic surgeons can accurately grade chondral lesions of the knee with the Outerbridge classification [19]. It is a morphological scoring system that directly reflects the changes in articular cartilage. In this study, we found that HELIX-II concentrations in synovial fluid change with cartilage damage. The content of HELIXII in the knee synovial fluid was low when the knee joint's cartilage surface was intact. The content of HELIX-II in the knee synovial fluid increased once cartilage damage occurred. This suggested that the synovial concentration of HELIX-II could be used as a biomarker for early damage of articular cartilage. Statistical analysis indicates that the synovial HELIX-II levels in knee joint are directly correlated with both Outerbridge cumulative scores and maximum scores.

Our study did not find a significant difference in the synovial HELIX-II level between Outerbridge scores 0 and 1 (slightly damaged cartilage), which indicates that the level of the synovial HELIX-II does not change for an intact cartilage surface. Surprisingly, we found no significant difference in the HELIX-II levels among the severely damaged cartilage sub-groups (Outerbridge scores of 2, 3, and 4 points), but we did find a significant difference between the slightly damaged and severely damaged cartilage groups. As the Outerbridge score of 1 only refers to softening and swelling of articular cartilage, while Outerbridge scores between 2 and 4 points indicate the rupture of the cartilage surface, this finding implies that once the surface of cartilage is fissured, the levels of HELIX-II in the synovial fluid increase sharply. The detailed mechanism remains unclear. One of the possible reasons is that the intact surface of the articular cartilage may have worked as a barrier for the release of collagen metabolites, and that HELIX-II is only released into the synovial fluid when fissures form on the cartilage surface, or flakes are detached from the surface. 
There are several possible explanations for the absence of differences in the synovial HELIX-II levels among Outerbridge 2-, 3-, and 4-point sub-groups. One of the possible explanations is that there is less type II collagen to be degenerated and released into synovial fluid when the cartilage damage becomes more severe and cartilage is lost. The second possible explanation may be different enzymatic pathway activities at the different stages of cartilage degradation. A recent study indicated that the type II fragments Helix-II and CTX-II are generated in part by different enzymatic pathways including cathepsins and matrix metalloproteases [9]. It would be interesting to explore whether the observations in this study are due to a different enzymatic activity at the different stages of cartilage damage.

In conclusion, the level of the HELIX-II in the knee synovial fluid could be used as an early biomarker to reflect cartilage damage, but cannot predict the severity and progression of knee articular cartilage damage.

Acknowledgments This project was supported by NIH AR052479 and 1R01AR059142-01A1, and by grants from the Aircast Foundation, Arthritis National Research Foundation, NSF 81071495 and 81171676, and SXNSF 2011011042. The authors gratefully acknowledge Ericka M. Bueno, Ph.D. for help with the manuscript preparation and editorial services.

Conflict of interest All authors declare there are no conflicts of interest regarding specific financial interests that are relevant to the work conducted or reported in this manuscript.

Open Access This article is distributed under the terms of the Creative Commons Attribution Noncommercial License which permits any noncommercial use, distribution, and reproduction in any medium, provided the original author(s) and source are credited.

\section{References}

1. Radin EL, Rose RM (1986) Role of subchondral bone in the initiation and progression of cartilage damage. Clin Orthop Relat Res (213):34-40

2. Wei L, Svensson O, Hjerpe A (1997) Correlation of morphologic and biochemical changes in the natural history of spontaneous osteoarthrosis in guinea pigs. Arthr Rheum 40(11):2075-2083

3. Thomas E, Peat G, Mallen C, Wood L, Lacey R, Duncan R, Croft P (2008) Predicting the course of functional limitation among older adults with knee pain: do local signs, symptoms and radiographs add anything to general indicators? Ann Rheum Dis 67(10):1390-1398

4. Wood L, Peat G, Thomas E, Hay EM, Sim J (2008) Associations between physical examination and self-reported physical function in older community-dwelling adults with knee pain. Phys Ther 88(1):33-42

5. Hinton R, Moody RL, Davis AW, Thomas SF (2002) Osteoarthritis: diagnosis and therapeutic considerations. Am Family Phys 65(5):841-848

6. Wei L, Fleming BC, Sun X, Teeple E, Wu W, Jay GD, Elsaid KA, Luo J, Machan JT, Chen Q (2010) Comparison of differential biomarkers of osteoarthritis with and without posttraumatic injury in the Hartley guinea pig model. J Orthop Res 28(7):900-906

7. Pelletier JP, McCollum R, Cloutier JM, Martel-Pelletier J (1995) Synthesis of metalloproteases and interleukin 6 (IL-6) in human osteoarthritic synovial membrane is an IL-1 mediated process. J Rheum Suppl 43:109-114

8. Murphy G, Lee MH (2005) What are the roles of metalloproteinases in cartilage and bone damage? Ann Rheum Dis 64(Suppl 4):iv44-iv47

9. Charni-Ben Tabassi N, Desmarais S, Bay-Jensen AC, Delaisse JM, Percival MD, Garnero P (2008) The type II collagen fragments Helix-II and CTX-II reveal different enzymatic pathways of human cartilage collagen degradation. Osteoarthr Cartil 16(10):1183-1191

10. Gertz BJ, Shao P, Hanson DA, Quan H, Harris ST, Genant HK, Chesnut CH, Eyre DR (1994) Monitoring bone resorption in early postmenopausal women by an immunoassay for cross-linked collagen peptides in urine. J Bone Miner Res 9:135-142

11. Rorvik AM, Grondahl AM (1995) Markers of osteoarthritis: a review of the literature. Vet Surg 24:255-262

12. Elsaid KA, Jay GD, Chichester CO (2003) Detection of collagen type II and proteoglycans in the synovial fluids of patients diagnosed with non-infectious knee joint synovitis indicates early damage to the articular cartilage matrix. Osteoarthr Cartil 11(9):673-680

13. Garnero P, Charni N, Juillet F, Conrozier T, Vignon E (2006) Increased urinary type II collagen helical and C telopeptide levels are independently associated with a rapidly destructive hip osteoarthritis. Ann Rheum Dis 65(12):1639-1644

14. Charni N, Juillet F, Garnero P (2005) Urinary type II collagen helical peptide (HELIX-II) as a new biochemical marker of cartilage degradation in patients with osteoarthritis and rheumatoid arthritis. Arthr Rheum 52(4):1081-1090

15. Quintana DJ, Garnero P, Huebner JL, Charni-Ben Tabassi N, Kraus VB (2008) PIIANP and HELIXII diurnal variation. Osteoarthr Cartil 16(10):1192-1195

16. Bedson J, Croft PR (2008) The discordance between clinical and radiographic knee osteoarthritis: a systematic search and summary of the literature. BMC Musculoskelet Disord 9:116

17. Wei L, Hjerpe A, Brismar BH, Svensson O (2001) Effect of load on articular cartilage matrix and the development of guinea-pig osteoarthritis. Osteoarthr Cartil 9(5):447-453

18. Elsaid KA, Chichester CO (2006) Review: collagen markers in early arthritic diseases. Clinica Chimica Acta 365(1-2):68-77

19. Cameron ML, Briggs KK, Steadman JR (2003) Reproducibility and reliability of the Outerbridge classification for grading chondral lesions of the knee arthroscopically. Am J Sports Med 31(1):83-86 